\title{
The Young's Interference Experiment in the Light of the Single-photon Modeling of the Laser Radiation
}

\author{
A.P. Davydov \\ Dept. of Applied and Theoretical Physics of Physical- \\ Mathematical Faculty
}

Nosov Magnitogorsk State Technical University

Magnitogorsk, Russia

E-mail: ap-dav@yandex.ru

\author{
T.P. Zlydneva \\ Dept. of Applied Mathematics and Informatics of Physical- \\ Mathematical Faculty
}

E-mail: tapazl@yandex.ru

\begin{abstract}
The basic principles of quantum mechanics of the photon, describing its single-particle states by means of the wave function in the coordinate representation are given. This wave function is a wave packet constructed by the superposition of the basic bivectors which are eigenfunctions of the operators of energy, momentum and helicity. The results of modeling in the space-time of such wave packet with a Gaussian distribution on the momentum of the photon corresponding femtosecond laser radiation are discussed. On the basis of the general ideas about the evolution of this packet and within the framework of the constructed photon quantum mechanics the quantum-mechanical approach is proposed to explain the wave phenomena exhibited by light such as the light interference in Young's experiment. It is emphasized that actually the photon isn't some "created" quantum corpuscle, but it's a quasi-particle that is a result of the propagation of spin waves in physical vacuum, the nature of which should be considered at the Planck distances simultaneously with the structure of the leptons and other fundamental particles.
\end{abstract}

Keywords-Schrödinger equation; Maxwell's equations; wave packet; probability density; bivector; detector; extreme maximon; Planck's parameters; wave-particle duality.

\section{INTRODUCTION}

It is known that the light and also the microparticles having mass exhibit similar corpuscular and wave properties. However, if for example for electrons these properties can be explained at least formally by means of wave function in coordinate representation, within usual quantum mechanics (without secondary quantization), then for photons the diffraction and interference are still explained in the language of classical electrodynamics. The purpose of this paper is to make the first step in the direction to correct this situation. Namely, based on illustrative results of the previous [1-5] modeling of the space-time propagation of the photon wave packet corresponding to a short-pulse laser radiation, we will offer below an explanation of the occurrence of an interference pattern with single photons in the experiment of type of the two-slit interference Young's experiment, having applied with respect to the photon the concept of wave function in coordinate representation. Nevertheless, we will specify further that this concept characterizes the photon only conditionally, owing to the fact that it still can't be considered as the certain created particle-corpuscle. In our opinion, the photon propagation is a consequence of excitation and propagation in space of some excited quantum state of physical vacuum. First of all, the excitation in the vacuum of certain spin wave like a magnon in a solid body is most probable. Although the nature of the corresponding excitation can be quite complex as it is evidenced e. g. by the manifestation of vector dominance in the interaction of photon with hadrons through strong interaction.

\section{Photon Wave Function in Coordinate REPRESENTATION}

For a long time it was considered [6-13] that the photon wave function can not be built in configuration space although in momentum representation it is applied in many areas. The reason for this is the zero ("rest") mass of the photon. In modern experiments (transfer of optical signals on quantum communication channels, "quantum teleportation", "paradoxes" with single photons, the problems of spatial entanglement and quantum computing, etc.) there is a need for association of photons with the localized carriers of elementary units of information. Therefore the building of photon wave function in coordinate representation becomes again actual "at the new level of knowledge". Then, knowing the wave function, it is also possible with the quantum-mechanical point of view to explain the interference, diffraction and polarization of electromagnetic waves. Without the full review here, we will refer on [7-19] where anyway this subject is touched, the term "wave function of a photon" is used, but nevertheless wave function of a photon, normalized on unit probability, isn't given in coordinate representation. Apparently, the first works in which the idea of a possible photon "localization" described by a probability density, defined by the normalized per unit wave function, has been proposed are [20-23]. Further development of the theory and justification of building of coordinate single-particle wave function of the photon was performed in [24-32] and others.

According to $[5,27,32]$ the photon wave function in coordinate representation has the following form: 


$$
\begin{aligned}
& \Psi^{( \pm)}(\mathbf{r}, t)=\int b(\mathbf{k}, \pm 1) \frac{\mathrm{e}_{ \pm 1}(\mathbf{k})}{(2 \pi)^{3 / 2}} e^{i(\mathbf{k r} \mp k c t)}\left(\begin{array}{l}
1 \\
0
\end{array}\right) d^{3} \mathbf{k}+ \\
& +\int[b(-\mathbf{k}, \mp 1)]^{*} \frac{\mathrm{e}_{\mp 1}(\mathbf{k})}{(2 \pi)^{3 / 2}} e^{i(\mathbf{k r} \mp k c t)}\left(\begin{array}{l}
0 \\
1
\end{array}\right) d^{3} \mathbf{k},
\end{aligned}
$$

where the top sign of all indexes corresponds to positive energy of photon, and the lower sign answers to negative energy, hypothetically possible; coefficients $b(\mathbf{k}, \lambda)$ are basically arbitrary, but if the photon state initially set by means of the electric (E) and magnetic $(\mathbf{H})$ fields intensities (in Gaussian System $), b(\mathbf{k}, \lambda)$ are expressed through them, as well as they satisfy to the normalization condition for the wave packet (1):

$$
\begin{gathered}
\left\langle\Psi^{( \pm)} \mid \Psi^{( \pm)}\right\rangle \equiv \int d^{3} \mathbf{r}\left[\Psi^{( \pm)}(\mathbf{r}, t)\right]^{+} \Psi^{( \pm)}(\mathbf{r}, t) \equiv \int d^{3} \mathbf{r} \rho_{P}^{( \pm)}(\mathbf{r}, t)= \\
=\int d^{3} \mathbf{k}\left[\Psi^{( \pm)}(\mathbf{k}, t)\right]^{+} \Psi^{( \pm)}(\mathbf{k}, t) \equiv \int d^{3} \mathbf{k} \rho_{P}^{( \pm)}(\mathbf{k})=1 .
\end{gathered}
$$

Here photon detection probability density in coordinate and momentum space (more precisely in the wave vector $\mathbf{k}=\mathbf{p} / \hbar$ space, where $\mathbf{p}$ is photon momentum), respectively is equal to

$$
\begin{gathered}
\rho_{P}^{( \pm)}(\mathbf{r}, t)=\left[\Psi^{( \pm)}(\mathbf{r}, t)\right]^{+} \Psi^{( \pm)}(\mathbf{r}, t), \\
\rho_{P}^{( \pm)}(\mathbf{k}, t)=\left[\Psi^{( \pm)}(\mathbf{k}, t)\right]^{+} \Psi^{( \pm)}(\mathbf{k}, t),
\end{gathered}
$$

moreover, in accordance with (1) the photon wave function in momentum representation is equal to

$$
\begin{gathered}
\Psi^{( \pm)}(\mathbf{k}, t) \equiv \frac{1}{(2 \pi)^{3 / 2}} \int e^{-i \mathbf{k r}} \Psi^{( \pm)}(\mathbf{r}, t) d^{3} \mathbf{r}= \\
=e^{\mp i k c t}\left\{b(\mathbf{k}, \pm 1) e_{ \pm 1}(\mathbf{k})\left(\begin{array}{l}
1 \\
0
\end{array}\right)+[b(-\mathbf{k}, \mp 1)]^{*} e_{\mp 1}(\mathbf{k})\left(\begin{array}{l}
0 \\
1
\end{array}\right)\right\} .
\end{gathered}
$$

Therefore photon detection probability density in the momentum space actually doesn't depend on time and it is

$$
\rho_{P}^{( \pm)}(\mathbf{k})=|b(\mathbf{k}, \pm 1)|^{2}+|b(-\mathbf{k}, \mp 1)|=\sum_{\lambda}|b(\mathbf{k}, \lambda)|^{2}
$$

where $\lambda= \pm 1$ takes two possible values of the photon helicity.

Equation (7) follows from (4) - (6), taking into account the properties of orthonormality of the complex polarization vectors

$$
\mathbf{e}_{\lambda}(\mathbf{k})=\left[\mathbf{e}_{I}(\mathbf{k})+i \lambda \mathbf{e}_{I I}(\mathbf{k})\right] / \sqrt{2}
$$

namely

$$
\left(\mathbf{e}_{\lambda^{\prime}}^{*} \mathbf{e}_{\lambda}\right)=\delta_{\lambda^{\prime} \lambda} ; \quad \mathrm{e}_{\lambda^{\prime}}^{+} \mathrm{e}_{\lambda}=\delta_{\lambda^{\prime} \lambda}
$$

In addition, with $\mathbf{n}=\mathbf{k} / k$ the following relations take place:

$$
\begin{gathered}
\mathbf{e}_{\lambda}(\mathbf{n})=\mathbf{e}_{-\lambda}(-\mathbf{n}) ; \quad\left[\mathbf{e}_{\lambda}(\mathbf{k})\right]^{*}=\mathbf{e}_{-\lambda}(\mathbf{k})=\mathbf{e}_{\lambda}(-\mathbf{k}), \\
\left|\mathbf{e}_{I}\right|=\left|\mathbf{e}_{I I}\right|=1 ; \quad\left(\mathbf{e}_{I} \mathbf{n}\right)=\left(\mathbf{e}_{I I} \mathbf{n}\right)=\left(\mathbf{e}_{I} \mathbf{e}_{I I}\right)=0, \\
\mathbf{e}_{I}(\mathbf{n})=\mathbf{e}_{I}(-\mathbf{n}) ; \quad \mathbf{e}_{I I}=\left\lfloor\mathbf{n} \times \mathbf{e}_{I}\right], \\
\mathbf{n}=\left[\mathbf{e}_{I} \times \mathbf{e}_{I I}\right]=i \lambda\left[\mathbf{e}_{\lambda} \times \mathbf{e}_{\lambda}^{*}\right]=\lambda \mathrm{e}_{\lambda}^{+}(\mathbf{k}) \hat{\mathbf{s}} \mathrm{e}_{\lambda}(\mathbf{k}),
\end{gathered}
$$

where $\hat{\mathbf{s}}$ is the operator of the photon spin in vector representation:

$$
\begin{gathered}
\hat{\mathbf{s}}=\mathbf{e}_{x} \hat{\mathbf{s}}_{x}+\mathbf{e}_{y} \hat{\mathbf{s}}_{y}+\mathbf{e}_{z} \hat{\mathbf{s}}_{z}= \\
=\mathbf{e}_{x}\left(\begin{array}{ccc}
0 & 0 & 0 \\
0 & 0 & -i \\
0 & i & 0
\end{array}\right)+\mathbf{e}_{y}\left(\begin{array}{ccc}
0 & 0 & i \\
0 & 0 & 0 \\
-i & 0 & 0
\end{array}\right)+\mathbf{e}_{z}\left(\begin{array}{ccc}
0 & -i & 0 \\
i & 0 & 0 \\
0 & 0 & 0
\end{array}\right)=i\left(\begin{array}{ccc}
0 & -\mathbf{e}_{z} \mathbf{e}_{y} \\
\mathbf{e}_{z} & 0 & -\mathbf{e}_{x} \\
-\mathbf{e}_{y} & \mathbf{e}_{x} & 0
\end{array}\right),(15
\end{gathered}
$$

where $\mathbf{e}_{x}, \mathbf{e}_{y}, \mathbf{e}_{z}$ are the basis vectors of $x, y, z$ axes.

The photon wave function $\Psi^{( \pm)}(\mathbf{r}, t)$ in coordinate representation satisfies to the equation of the Schrödinger equation type

$$
i \hbar \frac{\partial \Psi^{( \pm)}(\mathbf{r}, t)}{\partial t}=\hat{H}_{\mathrm{bv}} \Psi^{( \pm)}(\mathbf{r}, t)
$$

where

$$
\hat{H}_{\mathrm{bv}}=c\left(\hat{\boldsymbol{\alpha}}_{\mathrm{bv}} \hat{\mathbf{p}}\right)=\frac{c}{s}\left(\begin{array}{cc}
(\hat{\mathbf{s}} \hat{\mathbf{p}}) & 0 \\
0 & -(\hat{\mathbf{s}} \hat{\mathbf{p}})
\end{array}\right)
$$

is the Hamiltonian operator of free photon (having spin $s=1$ ) in bivector representation, in which its spin vector operator is equal to

$$
\hat{\mathbf{S}}=\mathbf{e}_{x} \hat{\mathrm{S}}_{x}+\mathbf{e}_{y} \hat{\mathrm{S}}_{y}+\mathbf{e}_{z} \hat{\mathrm{S}}_{z}=\left(\begin{array}{cc}
\hat{\mathbf{s}} & 0 \\
0 & \hat{\mathbf{s}}
\end{array}\right)
$$

$\hat{\mathbf{p}}=-i \hbar \hat{\nabla}$ is particle momentum operator; the matrix $\hat{\alpha}_{\mathrm{bv}}$ in bivector representation has form

$$
\hat{\boldsymbol{\alpha}}_{\mathrm{bv}}=\left(\begin{array}{cc}
\hat{\mathbf{s}} & 0 \\
0 & -\hat{\mathbf{s}}
\end{array}\right)
$$

Equation (16) is similar to the equation, which is satisfied by bivector $\Phi_{\mathrm{bv}}=\left(\begin{array}{l}\xi \\ \eta\end{array}\right)$, with which it is also possible to describe [12] the photon state in the coordinate representation. Here the physical quantities $\xi$ and $\eta$ (in matrix form) are

$$
\xi=\left(\begin{array}{c}
\mathrm{E}_{x}+i \mathrm{H}_{x} \\
\mathrm{E}_{y}+i \mathrm{H}_{y} \\
\mathrm{E}_{z}+i \mathrm{H}_{z}
\end{array}\right) ; \quad \eta=\left(\begin{array}{c}
\mathrm{E}_{x}-i \mathrm{H}_{x} \\
\mathrm{E}_{y}-i \mathrm{H}_{y} \\
\mathrm{E}_{z}-i \mathrm{H}_{z}
\end{array}\right),
$$

but they are independent quantities from each other [12]. The bivector $\Phi_{b v}$ satisfies to the equation of type (16):

$$
i \hbar \frac{\partial \Phi_{\mathrm{bv}}{ }^{( \pm)}(\mathbf{r}, t)}{\partial t}=\hat{H}_{\mathrm{bv}} \Phi_{\mathrm{bv}}{ }^{( \pm)}(\mathbf{r}, t)
$$


which is the consequence $[5,27,32]$ of the Maxwell equations written in the Majorana form [33, 12]

$i \hbar \frac{\partial \xi}{\partial t}=c(\hat{\mathbf{s}} \hat{\mathbf{p}}) \xi ; i \hbar \frac{\partial \eta}{\partial t}=-c(\hat{\mathbf{s}} \hat{\mathbf{p}}) \eta ;(\hat{\mathbf{p}} \xi)=0 ;(\hat{\mathbf{p}} \eta)=0$.

Four independent solutions of the equation (21) which are automatically satisfying to the equations (22), and also being eigenfunctions of the helicity operator

$$
\hat{\Lambda}=\frac{(\hat{\mathbf{S}} \hat{\mathbf{p}})}{s p}=\frac{(\hat{\mathbf{S}} \hat{\mathbf{p}})}{p}=\frac{1}{p}\left(\begin{array}{cc}
(\hat{\mathbf{s}} \hat{\mathbf{p}}) & 0 \\
0 & (\hat{\mathbf{s}} \hat{\mathbf{p}})
\end{array}\right)
$$

(in bivector representation) and the generalized eigenfunctions of the momentum operator, are the following [5, 27, 32]:

1) Corresponding to states of a photon with positive energy $E^{(+)}(k)=\hbar k c=+p c$ (wich are consistent with the special theory of relativity [34]) the orthonormal bivectors, answering to a helicity $\lambda= \pm 1$, are

$$
\begin{aligned}
& \Phi_{\mathrm{bv} ; \mathbf{k},+1}^{(+)}(\mathbf{r}, t)=\left(\begin{array}{c}
\xi_{\mathbf{k},+1}^{(+)}(\mathbf{r}, t) \\
0
\end{array}\right)=\frac{(\mathrm{Oe}) \mathrm{e}_{+1}(\mathbf{k})}{(2 \pi)^{3 / 2}} e^{i(\mathbf{k r}-k c t)}\left(\begin{array}{l}
1 \\
0
\end{array}\right), \\
& \Phi_{\mathrm{bv} ; \mathbf{k},-1}^{(+)}(\mathbf{r}, t)=\left(\begin{array}{c}
0 \\
\eta_{\mathbf{k},-1}^{(+)}(\mathbf{r}, t)
\end{array}\right)=\frac{(\mathrm{Oe}) \mathrm{e}_{-1}(\mathbf{k})}{(2 \pi)^{3 / 2}} e^{i(\mathbf{k r}-k c t)}\left(\begin{array}{l}
0 \\
1
\end{array}\right),
\end{aligned}
$$

respectively, where (Oe) is unit of measure (Oersted) of values $\xi$ and $\eta$.

2) Corresponding to states of a photon with negative energy $E^{(-)}(k)=-\hbar k c=-p c$ (which are theoretically possible) the orthonormal bivectors, answering to the helicity $\lambda=\mp 1$, are

$$
\begin{aligned}
& \Phi_{\mathrm{bv} ; \mathbf{k},-1}^{(-)}(\mathbf{r}, t)=\left(\begin{array}{c}
\xi_{\mathbf{k},-1}^{(-)}(\mathbf{r}, t) \\
0
\end{array}\right)=\frac{(\mathrm{Oe}) \mathrm{e}_{-1}(\mathbf{k})}{(2 \pi)^{3 / 2}} e^{i(\mathbf{k r}+k c t)}\left(\begin{array}{l}
1 \\
0
\end{array}\right), \\
& \Phi_{\mathrm{bv} ; \mathbf{k},+1}^{(-)}(\mathbf{r}, t)=\left(\begin{array}{c}
0 \\
\eta_{\mathbf{k},+1}^{(-)}(\mathbf{r}, t)
\end{array}\right)=\frac{(\mathrm{Oe}) \mathrm{e}_{+1}(\mathbf{k})}{(2 \pi)^{3 / 2}} e^{i(\mathbf{k r}+k c t)}\left(\begin{array}{l}
0 \\
1
\end{array}\right)
\end{aligned}
$$

Taking into account (9), the basis vectors $\xi_{\mathbf{k}, \lambda}(\mathbf{r}, t)$ and $\eta_{\mathbf{k}, \lambda}(\mathbf{r}, t)$, also being in turn eigenfunctions of operators of the energy, momentum and helicity $\hat{\lambda}=(\hat{\mathbf{s}} \hat{\mathbf{p}}) / p$ (in vector representation), satisfy to the orthonormality relations

$$
\begin{aligned}
& \int d^{3} \mathbf{r} \xi_{\mathbf{k}^{\prime}, \lambda^{\prime}}^{+}(\mathbf{r}, t) \xi_{\mathbf{k}, \lambda}(\mathbf{r}, t)=\delta_{\lambda^{\prime} \lambda} \delta\left(\mathbf{k}^{\prime}-\mathbf{k}\right)(\mathrm{Oe})^{2}, \\
& \int d^{3} \mathbf{r} \eta_{\mathbf{k}^{\prime}, \lambda^{\prime}}^{+}(\mathbf{r}, t) \eta_{\mathbf{k}, \lambda}(\mathbf{r}, t)=\delta_{\lambda^{\prime} \lambda} \delta\left(\mathbf{k}^{\prime}-\mathbf{k}\right)(\mathrm{Oe})^{2}
\end{aligned}
$$

Therefore bivectors (24) - (27) satisfy to the orthonormality relations

$\int d^{3} \mathbf{r}\left[\Phi_{\mathrm{bv} ; \mathbf{k}^{\prime}, \lambda^{\prime}}^{( \pm)}(\mathbf{r}, t)\right]^{+} \Phi_{\mathrm{bv} ; \mathbf{k}, \lambda}^{( \pm)}(\mathbf{r}, t)=(\mathrm{Oe})^{2} \delta_{\lambda^{\prime} \lambda} \delta\left(\mathbf{k}^{\prime}-\mathbf{k}\right)$.
In addition, bivectors corresponding to the states with the opposite energy sign are orthogonal to each other regardless of the values $\mathbf{k}$ and $\lambda$ :

$$
\int d^{3} \mathbf{r}\left[\Phi_{\mathrm{bv} ; \mathbf{k}^{\prime}, \lambda^{\prime}}^{( \pm)}(\mathbf{r}, t)\right]^{+} \Phi_{\mathrm{bv} ; \mathbf{k}, \lambda}^{(\mp)}(\mathbf{r}, t)=0 .
$$

The relations (28) - (30) make it possible to decompose any vectors and bivectors in the corresponding bases:

$$
\begin{aligned}
& \xi(\mathbf{r}, t)=\mathrm{E}(\mathbf{r}, t)+i \mathrm{H}(\mathbf{r}, t) \equiv \xi_{+1}^{(+)}(\mathbf{r}, t)+\xi_{-1}^{(-)}(\mathbf{r}, t)= \\
= & \int B(\mathbf{k},+1) \xi_{\mathbf{k},+1}^{(+)}(\mathbf{r}, t) d^{3} \mathbf{k}+\int B(\mathbf{k},-1) \xi_{\mathbf{k},-1}^{(-)}(\mathbf{r}, t) \\
& \eta(\mathbf{r}, t)=\mathrm{E}(\mathbf{r}, t)-i \mathrm{H}(\mathbf{r}, t) \equiv \eta_{-1}^{(+)}(\mathbf{r}, t)+\eta_{+1}^{(-)}(\mathbf{r}, t)= \\
= & \int[B(-\mathbf{k},-1)]^{*} \eta_{\mathbf{k},-1}^{(+)}(\mathbf{r}, t) d^{3} \mathbf{k}+\int[B(-\mathbf{k},+1)]^{*} \eta_{\mathbf{k},+1}^{(-)}(\mathbf{r}, t) d^{3} \mathbf{k}, \\
& \Phi_{\mathrm{bv}}^{( \pm)}(\mathbf{r}, t)=\left(\begin{array}{l}
\mathrm{E}_{\xi, \pm 1}^{( \pm)}(\mathbf{r}, t)+i \mathrm{H}_{\xi, \pm 1}^{( \pm)}(\mathbf{r}, t) \\
\mathrm{E}_{\eta, \mp 1}^{( \pm)}(\mathbf{r}, t)-i \mathrm{H}_{\eta, \mp 1}^{( \pm)}(\mathbf{r}, t)
\end{array}\right) \equiv\left(\begin{array}{c}
\xi_{ \pm 1}^{( \pm)}(\mathbf{r}, t) \\
\eta_{\mp 1}^{( \pm)}(\mathbf{r}, t)
\end{array}\right)= \\
= & \int B(\mathbf{k}, \pm 1) \Phi_{\mathrm{bv} ; \mathbf{k}, \pm 1}^{( \pm)}(\mathbf{r}, t) d^{3} \mathbf{k}+\int[B(-\mathbf{k}, \mp 1)]^{*} \Phi_{\mathrm{bv} ; \mathbf{k}, \mp 1}^{( \pm)}(\mathbf{r}, t) d^{3} \mathbf{k}=
\end{aligned}
$$

From (32) - (35) it is visible that if the free electromagnetic field is initially set by means of the some classically interpreted field intensities $\mathbf{E}$ and $\mathbf{H}$, the single-photon state corresponding to this field can't be generally specified in terms of quantities $\xi$ and $\eta$, as the unobserved negative energy make a contribution in decompositions (32) - (35).

So, at the level of the postulate we can say that the singlephoton state can be described bivector (36) - (37) with a plus sign for the real photons and with the minus sign for the hypothetical photons with negative energy.

The bivector (36) - (37) also plays an important role in the description of the single-photon state.

We can denote the intensities, giving the contributions to the integrals (32) - (35) according to the following relations:

$$
\begin{aligned}
& \mathrm{E}(\mathbf{r}, t)=\mathrm{E}_{\xi,+1}^{(+)}(\mathbf{r}, t)+\mathrm{E}_{\xi,-1}^{(-)}(\mathbf{r}, t)=\mathrm{E}_{\eta,-1}^{(+)}(\mathbf{r}, t)+\mathrm{E}_{\eta,+1}^{(-)}(\mathbf{r}, t), \\
& \mathrm{H}(\mathbf{r}, t)=\mathrm{H}_{\xi,+1}^{(+)}(\mathbf{r}, t)+\mathrm{H}_{\xi,-1}^{(-)}(\mathbf{r}, t)=\mathrm{H}_{\eta,-1}^{(+)}(\mathbf{r}, t)+\mathrm{H}_{\eta,+1}^{(-)}(\mathbf{r}, t) .
\end{aligned}
$$

Then from (36), (38) important connections follow:

$$
\begin{aligned}
& \mathrm{E}_{\xi, \mp 1}^{(\mp)}(\mathbf{r}, t)=\mathrm{E}_{\eta, \mp 1}^{( \pm)}(\mathbf{r}, t), \quad \mathrm{H}_{\xi, \mp 1}^{(\mp)}(\mathbf{r}, t)=\mathrm{H}_{\eta, \mp 1}^{( \pm)}(\mathbf{r}, t), \\
& {\left[\xi_{-1}^{(-)}(\mathbf{r}, t)\right]^{*}=\eta_{-1}^{(+)}(\mathbf{r}, t), \quad\left[\eta_{+1}^{(-)}(\mathbf{r}, t)\right]^{*}=\xi_{+1}^{(+)}(\mathbf{r}, t) .}
\end{aligned}
$$

Together with the formulas (43) - (44) associating with the decompositions (33), (35), the relations (40), (41) give [5, 27, 32] the superposition principle for the intensities $\mathbf{E}$ and $\mathbf{H}$ :

$$
\mathrm{E}(\mathbf{r}, t)=\mathrm{E}_{\xi,+1}^{(+)}(\mathbf{r}, t)+\mathrm{E}_{\eta,-1}^{(+)}(\mathbf{r}, t)
$$




$$
\mathrm{H}(\mathbf{r}, t)=\mathrm{H}_{\xi,+1}^{(+)}(\mathbf{r}, t)+\mathrm{H}_{\eta,-1}^{(+)}(\mathbf{r}, t) .
$$

Using specified bivectors, it is possible to write the spatial density distribution of photon energy in the state (37):

$$
\begin{gathered}
\rho_{E}^{( \pm)}(\mathbf{r}, t) \equiv \frac{1}{8 \pi}\left[\Phi_{\mathrm{bv}}^{( \pm)}(\mathbf{r}, t)\right]^{+} \Phi_{\mathrm{bv}}^{( \pm)}(\mathbf{r}, t)= \\
=\frac{1}{8 \pi}\left\{\left[\xi_{ \pm 1}^{( \pm)}(\mathbf{r}, t)\right]^{+} \xi_{ \pm 1}^{( \pm)}(\mathbf{r}, t)+\left[\eta_{\mp 1}^{( \pm)}(\mathbf{r}, t)\right]^{+} \eta_{\mp 1}^{( \pm)}(\mathbf{r}, t)\right\} \equiv \\
=\frac{1}{8 \pi}\left\{\left[\mathbf{E}_{\xi, \pm 1}^{( \pm)}(\mathbf{r}, t)\right]^{2}+\left[\mathbf{H}_{\xi, \pm 1}^{( \pm)}(\mathbf{r}, t)\right]^{2}+\left[\mathbf{E}_{\eta, \mp 1}^{( \pm)}(\mathbf{r}, t)\right]^{2}+\left[\mathbf{H}_{\eta, \mp 1}^{( \pm)}(\mathbf{r}, t)\right]^{2}\right\}
\end{gathered}
$$

This energy density is to be distinguished from the "classical" energy density, which, obviously, has the form

$$
\begin{gathered}
\rho_{E}^{(c l)}(\mathbf{r}, t) \equiv \frac{1}{8 \pi}\left\{[\mathbf{E}(\mathbf{r}, t)]^{2}+[\mathbf{H}(\mathbf{r}, t)]^{2}\right\}= \\
\equiv \frac{1}{16 \pi}\left[\Phi_{\mathrm{bv}}^{(+)}(\mathbf{r}, t)+\Phi_{\mathrm{bv}}^{(-)}(\mathbf{r}, t)\right]^{+}\left[\Phi_{\mathrm{bv}}^{(+)}(\mathbf{r}, t)+\Phi_{\mathrm{bv}}^{(-)}(\mathbf{r}, t)\right] .
\end{gathered}
$$

From the practical point of view, the construction of the bivector (36) - (37) isn't so much important in itself, how the finding of the coefficients $B(\mathbf{k}, \lambda)$ is important in the case, when the single-photon state is defined to be corresponding to the state of the electromagnetic field, initially given by means of the classical intensities $\mathbf{E}$ and $\mathbf{H}$. Further, composing, for example, the vector $\boldsymbol{\xi}=\mathbf{E}+i \mathbf{H}$ and using the orthonormality relations (28), (30), it is possible to calculate the coefficients

$$
\begin{gathered}
B(\mathbf{k}, \pm 1)=\frac{1}{(\mathrm{Oe})^{2}} \int d^{3} \mathbf{r}\left[\xi_{\mathbf{k}, \pm 1}^{( \pm)}(\mathbf{r}, t)\right]^{+} \xi(\mathbf{r}, t)= \\
=\frac{1}{(\mathrm{Oe})^{2}} \int d^{3} \mathbf{r}\left[\Phi_{\mathrm{bv} ; \mathbf{k}, \pm 1}^{( \pm)}(\mathbf{r}, t)\right]^{+} \Phi^{( \pm)}(\mathbf{r}, t) .
\end{gathered}
$$

Then with these coefficients we can calculate the coefficients $b(\mathbf{k}, \lambda)$ appearing in (1) by the formula

$$
b(\mathbf{k}, \lambda)=\frac{(\mathrm{Oe})}{\sqrt{8 \pi \hbar k c}} B(\mathbf{k}, \lambda) .
$$

The introduction of these coefficients most fully implements the principle of correspondence. Indeed, according to (7), (24) - (27), (34), (37), (47), based on the classical electrodynamics, the total photon energy can be written as

$$
\begin{aligned}
E & =E^{(+)}=\int d^{3} \mathbf{r} \rho_{E}^{(c l)}(\mathbf{r}, t)=\frac{(\mathrm{Oe})^{2}}{8 \pi} \int d^{3} \mathbf{k} \sum_{\lambda}|B(\mathbf{k}, \lambda)|^{2}= \\
& =\int d^{3} \mathbf{k} \sum_{\lambda}|b(\mathbf{k}, \lambda)|^{2} \hbar k c=\int d^{3} \mathbf{k} \rho_{P}^{( \pm)}(\mathbf{k}) \hbar k c .
\end{aligned}
$$

The integration of density $\rho_{E}^{( \pm)}(\mathbf{r}, t)$ yields just the same result.

On the other hand, the same result is obtained if we use the purely quantum-mechanical formula of calculating of the energy mean value in the state (1) and the Hamilton operator (17) (and also the equation (16), and relations (30)):

$$
\begin{aligned}
& \overline{E^{( \pm)}} \equiv\left\langle\Psi( \pm)\left|\hat{H}_{\mathrm{bv}}\right| \Psi^{( \pm)}\right\rangle=\left\langle\Psi^{( \pm)} \mid i \hbar \frac{\partial \Psi}{\partial t}\right\rangle= \\
= & \int( \pm \hbar k c) \sum_{\lambda}|b(\mathbf{k}, \lambda)|^{2} d^{3} \mathbf{k}=\int E^{( \pm)}(k) \rho_{P}^{( \pm)}(\mathbf{k}) d^{3} \mathbf{k}
\end{aligned}
$$

what coincides with (52) for positive energy.

To conclude this paragraph we mention that from the equation (16) the continuity equation follows [5, 27, 32] for density of probability $\rho_{P}^{( \pm)}(\mathbf{r}, t)$ and of stream density $\mathbf{j}_{P}^{( \pm)}(\mathbf{r}, t)$ of probability to find the photon in the vicinity of the point $\mathbf{r}$ in a time point $t$ :

$$
\frac{\partial \rho_{P}^{( \pm)}(\mathbf{r}, t)}{\partial t}+\operatorname{div} \mathbf{j}_{P}^{( \pm)}(\mathbf{r}, t)=0,
$$

where

$$
\begin{gathered}
\rho_{P}^{( \pm)}(\mathbf{r}, t)=\left[\Psi^{( \pm)}(\mathbf{r}, t)\right]^{+} \Psi^{( \pm)}(\mathbf{r}, t), \\
\mathbf{j}_{P}^{( \pm)}(\mathbf{r}, t)=c\left[\Psi^{( \pm)}(\mathbf{r}, t)\right]^{+} \hat{\boldsymbol{\alpha}}_{\mathrm{bv}} \Psi^{( \pm)}(\mathbf{r}, t) .
\end{gathered}
$$

Although for arbitrary coefficients $b(\mathbf{k}, \lambda)$ the equation (55) doesn't possess of relativistic invariance property, nevertheless it can be shown that this equation is relativistic invariant [27, 32] for the case of plane monochromatic waves.

\section{Modeling OF SHORT-Puls LASER RADiATiON}

On the basis of the above stated general method of construction of wave function of a free photon in coordinate representation in [1-4] the most important wave packet in scientific and methodical aspects with Gaussian momentum distribution is considered, namely with the coefficients

$$
\begin{gathered}
b(\mathbf{k}, \pm 1)=[b(-\mathbf{k}, \mp 1)]^{*}= \\
=\sqrt{\frac{\alpha^{3}}{2 \pi \sqrt{\pi}}} \exp \left[-\frac{\alpha^{2}}{2}\left(k_{x}^{2}+k_{y}^{2}+\left(k_{z} \mp k_{0}\right)^{2}\right)-i \mathbf{k} \mathbf{r}_{0}\right]
\end{gathered}
$$

where parameters $\quad \mathbf{k}_{0}=\left(0,0, k_{0}\right), \quad \mathbf{r}_{0}=\left(x_{0}, y_{0}, z_{0}\right), \quad \alpha$ characterize the average values and the dispersions of the corresponding physical quantities in the state of a photon (1) and satisfy the normalization condition (2).

Parameterization (58) answers to the state of a photon with zero average helicity as the $\lambda= \pm 1$ are presented in (58) with equal probability. All characteristics can be broken into two categories: momentum-energy and space-time. According to quantum mechanics, values of these categories characteristics answer to the corresponding uncertainty relations. New here, compared to the particles with mass, is the fact that the values of characteristics of the second category essentially can depend on "choice" of vectors $\mathbf{e}_{\lambda}(\mathbf{k})$. 


\section{A. Momentum-energy characteristics}

Applying (1), (58) and quantum-mechanical formula of calculation of average value of physical quantity $F$, the operator of which is equal $\hat{F}$, namely

$$
\overline{F^{( \pm)}} \equiv\left\langle\Psi^{( \pm)} \mid \hat{F} \Psi^{( \pm)}\right\rangle=\left\langle\sum_{\lambda^{\prime}} \Psi_{\lambda^{\prime}}^{( \pm)} \mid \hat{F} \sum_{\lambda} \Psi_{\lambda}^{( \pm)}\right\rangle,
$$

where $\Psi_{\lambda}^{( \pm)}$at the values $\lambda= \pm 1$ are defined by corresponding terms of the formula (1), we find at once average values of projections of momentum and their squares:

$$
\begin{gathered}
\overline{p_{x}^{( \pm)}}=\overline{p_{y}^{( \pm)}}=0, \overline{p_{z}^{( \pm)}}= \pm \hbar k_{0}, \\
\overline{\left(p_{x}^{( \pm)}\right)^{2}}=\overline{\left(p_{y}^{( \pm)}\right)^{2}}=\frac{\hbar^{2}}{2 \alpha^{2}}, \overline{\left(p_{z}^{( \pm)}\right)^{2}}=\frac{\hbar^{2}}{2 \alpha^{2}}+\hbar^{2} k_{0}^{2},
\end{gathered}
$$

from where it follows that the average vector of a momentum of the photon in a state of positive energy is directed along the axis $z$, and with negative is opposite to it:

$$
\overline{\mathbf{p}^{( \pm)}}= \pm \hbar \mathbf{k}_{0} \equiv \pm \hbar k_{0} \mathbf{e}_{z} .
$$

The dispersions of the projections of a momentum vector on the $x, y, z$ axes are defined by the parameter $\alpha$ :

$$
D_{p_{x}} \equiv \overline{\left(p_{x}^{( \pm)}\right)^{2}}-\left(\overline{p_{x}^{( \pm)}}\right)^{2}=\frac{\hbar^{2}}{2 \alpha^{2}}, D_{p_{y}}=D_{p_{z}}=\frac{\hbar^{2}}{2 \alpha^{2}} .
$$

Then uncertainty of the photon momentum projections in state (1) are reduced to formulas

$$
\Delta p_{x} \equiv \sqrt{D_{p_{x}}}=\frac{\hbar}{\alpha \sqrt{2}}, \quad \Delta p_{y}=\Delta p_{z}=\frac{\hbar}{\alpha \sqrt{2}},
$$

where the presence $\sqrt{2}$ is connected with such choice which gives, according to (6) the simplest form of the momentum distribution in the state (1), namely Gaussian form

$$
\begin{gathered}
\rho_{P}^{( \pm)}(\mathbf{k})=|b(\mathbf{k}, \pm 1)|^{2}+|b(-\mathbf{k}, \mp 1)|^{2}= \\
=\frac{\alpha^{3}}{\pi \sqrt{\pi}} \exp \left[-\alpha^{2}\left(k_{x}^{2}+k_{y}^{2}+\left(k_{z} \mp k_{0}\right)^{2}\right)\right] .
\end{gathered}
$$

Applying the formulas (54), (65), (66) we find the average energy of the photon in state (1), respectively with positive and negative spectrum of its energy,

$\overline{E^{( \pm)}}= \pm \hbar k_{0} c\left[\left(1+\frac{1}{2 \alpha^{2} k_{0}^{2}}\right) \operatorname{erf}\left(\alpha_{1} k_{0}\right)+\frac{\exp \left(-\alpha^{2} k_{0}^{2}\right)}{\alpha k_{0} \sqrt{\pi}}\right]$,

and also, similarly, the average square of energy of the photon in the state (1):

$$
\overline{\left(E^{( \pm)}\right)^{2}}=c^{2} \hbar^{2} k_{0}^{2}\left(1+\frac{3}{2 \alpha^{2} k_{0}^{2}}\right) .
$$

Using (67) and (68), it is possible to calculate dispersion and uncertainty of energy of a photon in state (1):

$$
D_{E}=\overline{\left(E^{( \pm)}\right)^{2}}-\left(\overline{E^{( \pm)}}\right)^{2}, \quad \Delta E=\sqrt{D_{E}} .
$$

\section{B. Space-time characteristics}

Requirements (8) - (13) are satisfied, e.g., for the following polarization vectors $[1,3-5]$ :

$$
\begin{aligned}
& e_{I}(\mathbf{k})=\left(\begin{array}{c}
1-(1-\cos \theta) \cos ^{2} \varphi \\
-(1-\cos \theta) \sin \varphi \cos \varphi \\
-\sin \theta \cos \varphi
\end{array}\right) \\
& e_{I I}(\mathbf{k})=\left(\begin{array}{c}
-(1-\cos \theta) \sin \varphi \cos \varphi \\
\cos \theta+(1-\cos \theta) \cos ^{2} \varphi \\
-\sin \theta \sin \varphi
\end{array}\right) \text { at } 0 \leq \theta \leq \frac{\pi}{2} \\
& e_{I}(\mathbf{k})=\left(\begin{array}{c}
1-(1+\cos \theta) \cos ^{2} \varphi \\
-(1+\cos \theta) \sin \varphi \cos \varphi \\
\sin \theta \cos \varphi
\end{array}\right), \\
& e_{I I}(\mathbf{k})=\left(\begin{array}{c}
(1+\cos \theta) \sin \varphi \cos \varphi \\
\cos \theta-(1+\cos \theta) \cos ^{2} \varphi \\
-\sin \theta \sin \varphi
\end{array}\right) \text { at } \frac{\pi}{2}<\theta \leq \pi
\end{aligned}
$$

where the Cartesian components of the corresponding vectors in the configuration space are specified, expressed in terms of the spherical coordinates of vector $\mathbf{k}$ in momentum space. Taking into account formulas (70) - (73) it is conveniently to carry out the calculation of space-time characteristics in momentum representation, using the formula (6).

In particular for the average values of coordinates and their squares of a point of detection of the photon in state (1) we obtain the following expressions:

$$
\begin{gathered}
\overline{x^{( \pm)}}=x_{0} ; \quad \overline{y^{( \pm)}}=y_{0} ; \quad \overline{z^{( \pm)}}=z_{0} \pm c t \overline{n_{z}^{( \pm)}}, \\
\overline{\left(x^{( \pm)}\right)^{2}}=x_{0}^{2}+\frac{\alpha^{2}}{2}+A_{1}^{(2)}+c^{2} t^{2} \overline{\left(n_{x}^{( \pm)}\right)^{2}}, \\
\overline{\left(y^{( \pm)}\right)^{2}}=y_{0}^{2}+\frac{\alpha^{2}}{2}+A_{2}^{(2)}+c^{2} t^{2} \overline{\left(n_{y}^{( \pm)}\right)^{2}}, \\
\overline{\left(z^{( \pm)}\right)^{2}}=z_{0}^{2}+\frac{\alpha^{2}}{2}+A_{3}^{(2)}+c^{2} t^{2} \overline{\left(n_{z}^{( \pm)}\right)^{2}} \pm 2 c t \overline{n_{z}^{( \pm)}} z_{0},
\end{gathered}
$$

where

$$
\begin{gathered}
\overline{n_{z}^{( \pm)}}=\left[\left(1-\frac{1}{2 \alpha^{2} k_{0}^{2}}\right) \operatorname{erf}\left(\alpha k_{0}\right)-\frac{\exp \left(-\alpha^{2} k_{0}^{2}\right)}{\alpha k_{0} \sqrt{\pi}}\right], \\
\overline{\left(n_{x}^{( \pm)}\right)^{2}}=\overline{\left(n_{y}^{( \pm)}\right)^{2}}=\frac{1}{2}\left[1-\overline{\left(n_{z}^{( \pm)}\right)^{2}}\right]=
\end{gathered}
$$




$$
\begin{gathered}
=\frac{1}{2 \alpha^{2} k_{0}^{2}}\left[1-\frac{\sqrt{\pi}}{2 \alpha k_{0}} \operatorname{erfi}\left(\alpha k_{0}\right) \exp \left(-\alpha^{2} k_{0}^{2}\right)\right], \\
A_{1}^{(2)}=A_{2}^{(2)}=-\frac{1}{2} A_{3}^{(2)}+\Delta A_{13}^{(2)}, \\
A_{3}^{(2)}=\frac{1}{2 k_{0}^{2}}\left[\frac{1+2 \alpha^{2} k_{0}^{2}}{2 \alpha k_{0}} \operatorname{erfi}\left(\alpha k_{0}\right) \exp \left(-\alpha^{2} k_{0}^{2}\right) \sqrt{\pi}-1\right], \\
\Delta A_{13}^{(2)} \equiv 2 \alpha^{2} \exp \left(-\alpha^{2} k_{0}^{2}\right) \int_{0}^{1} \frac{\exp \left(\alpha^{2} k_{0}^{2} u^{2}\right)}{1+u} d u .
\end{gathered}
$$

From (74) - (83) it follows that the dispersions $D_{x}, D_{y}, D_{z}$ of coordinates of detection point of the photon which is in the state (1) parameterized by means of (58) are equal

$$
\begin{gathered}
D_{x}=D_{y}=\frac{\alpha^{2}}{2}+A_{1}^{(2)}+c^{2} t^{2} \overline{\left(n_{x}^{( \pm)}\right)^{2}}=\frac{\alpha^{2}}{2}+A_{1}^{(2)}+c^{2} t^{2} D_{n_{x}} \\
D_{z}=\frac{\alpha^{2}}{2}+A_{3}^{(2)}+c^{2} t^{2} D_{n_{z}} .
\end{gathered}
$$

According to (84) the dispersions $D_{x}$ and $D_{y}$ are the same for the considered wave packet which is symmetric relatively of the $z$ axis.

\section{Analysis of modeling results}

As seen from (84), the rate of expansion of the wave packet is the same in each plane $x y$, in accordance with the fact that the wave packet (1) with the parameterization (58) remains symmetric relatively of the $\mathrm{z}$ axis. As characteristics of speed of this expansion it is possible to use periods $\tau_{x}, \tau_{y}$, $\tau_{z}$ during which initial dispersions (at $t=0$ ) are doubled along the directions $x, y, z$. From (84) we find:

$$
\tau_{x}=\frac{\Delta x(t=0)}{c \Delta n_{x}}=\tau_{y}=\frac{\Delta y(t=0)}{c \Delta n_{y}} ; \quad \tau_{z}=\frac{\Delta z(t=0)}{c \Delta n_{z}} .
$$

Since even in a simple form of distribution (58) it is not possible analytically to obtain an expression for the probability density in configuration space, we carry out the analysis of the evolution of the considered wave packet by means of calculation of the intensity of electric field, using the initial formula (1), (42), (51). Not equal to zero in this case the projection of intensity $E_{x}$ is only; it characterizes a certain way, the spatial probability density. The spatial "form" of a wave packet in the initial time is "spherical". We will give results of numerical calculation for the packet corresponding to the duration of radiation $\tau_{\text {rad }}=80 \mathrm{fs}$ with the central wavelength of 10 microns. Parameter $\alpha=0.001692$ was calculated using the uncertainty relation for energy and time $\Delta E \Delta t \geq \hbar / 2$, where $\Delta t$ was assumed equal to $\tau_{\text {rad }} / 2$, and $\Delta E$ was determined by the formulas (67) - (69).

On an axis of symmetry of a packet, the density of probability of photon detection in the vicinity of the center of the packet (with coordinate $z_{c}$ ) moves practically with velocity of light in vacuum. The farther from the axis, the lower the velocity of probability density is in the direction of the average velocity (along $z$ axis) of wave packet.
Thus, there is a transformation of the original shape of the initial wave packet (Fig. 1) to a certain "conical" shape (Fig. 2 and 3 ).

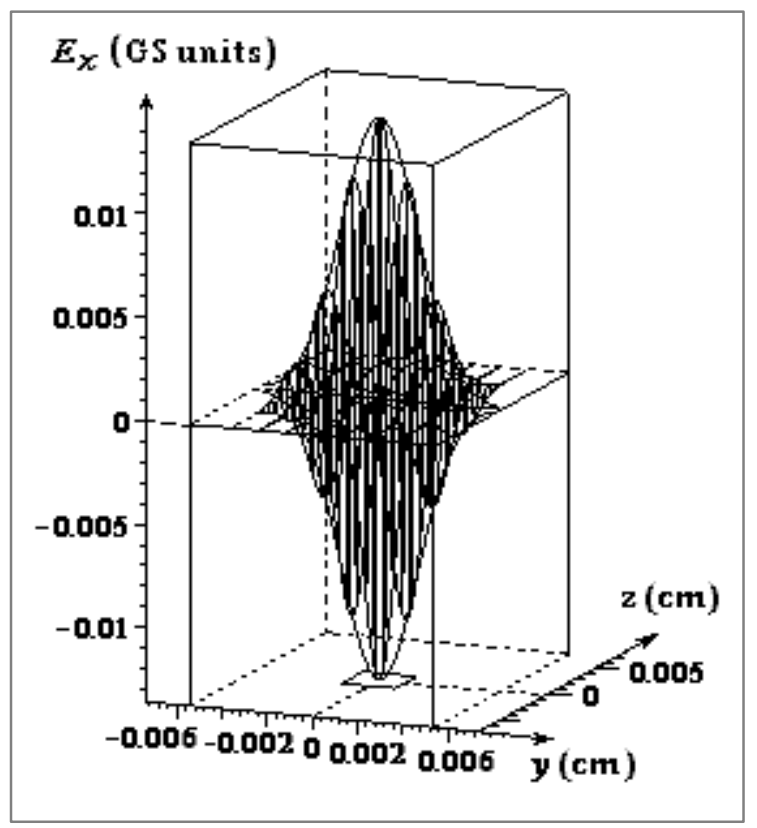

Fig. 1. Distribution of electric field intensity $E_{x}$ at time moment $t=0$ : $z_{c}=0, \Delta x=\Delta y=\Delta z=0,0120 \mathrm{~cm}$.

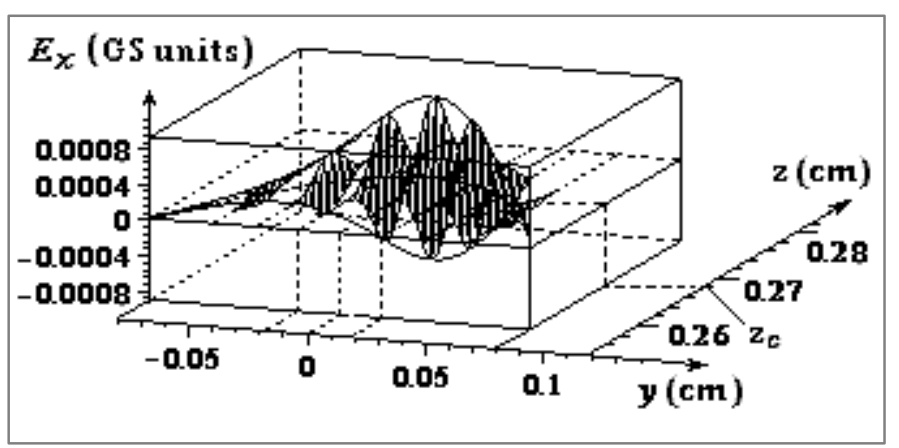

Fig. 2. Distribution of electric field intensity $E_{x}$ at time moment $t=\tau_{z}$ : $z_{c}=0,268 \mathrm{~cm}, \Delta x=\Delta y=0,0179 \mathrm{~cm}, \Delta z=0,00169 \mathrm{~cm}$.

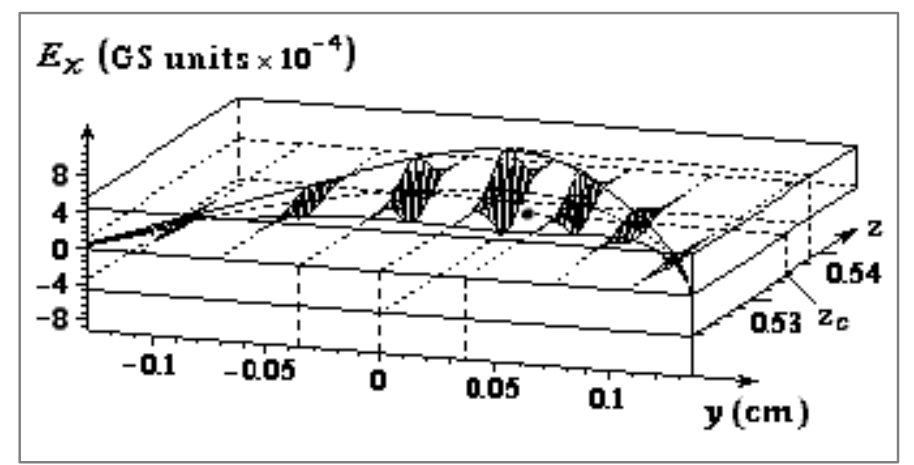

Fig. 3. Distribution of electric field intensity $E_{x}$ at time moment $t=2 \tau_{z}$ : $z_{c}=0,536 \mathrm{~cm}, \Delta x=\Delta y=0,0358 \mathrm{~cm}, \Delta z=0,00268 \mathrm{~cm}$. 
Speed of this transformation is the more, the less initial "radius" of a wave packet (1), according to the general representations of quantum mechanics. Fig. 1-3 show the distributions of the most significant projection of the intensity of electric field $E_{x}$ computed respectively in three different moments of time: $t=0, t=\tau_{z}=14,9 \tau_{y}=0,898 \cdot 10^{-11} \mathrm{c}$ and $t=2 \tau_{z}$, where $\tau_{z}$ is the time of expansion of the packet (85) along an $z$ axis.

\section{The Main Formula of WaVe-Particle Duality AND NATURE OF PHOTON}

In our opinion the constructed photon quantum mechanics substantially removes a problem of wave-particle duality of quantum "particles". Main "formula" of wave-particle duality of light and particles can be formulated as follows [5, 32, 35]:

1. Photons and microparticles at interaction behave as a corpuscles, transferring and transmitting (to other particles) in a certain quantity as dynamic characteristics (energy, momentum, angular momentum), and "internal" (mass, electric charge, spin, etc.). In particular, such transfer is carried out at hit of a photon or microparticle in quite dot detector (or a point on the screen) with coordinate $\mathbf{r}$ at time point $t$. The fact of hit of "all particle entirely" in the dot detector is characteristic for a corpuscle, but not for some real wave.

2. However photons and microparticles propagate in space by "wave rules", that is their distribution in space is described by the wave function. In particular, density of probability of detection in space of the nonrelativistic particle with a nonzero mass is postulated by a formula $\rho(\mathbf{r}, t)=|\Psi(\mathbf{r}, t)|^{2}$, and a photon by (3). This probability density also causes the hit of a photon and microparticle in the dot detector. A characteristic interferential picture on the screen corresponds to distribution of $\rho(\mathbf{r}, t)$ along the screen.

Nevertheless, electromagnetic radiation even in the case of waves small lengths and obviously expressed "corpuscular properties", is impossible to consider as a stream of the certain "created", "dot" particles, similar to the massive particles. In our view, the photon is a quasi-particle, and light is a result of the propagation of a spin wave in physical vacuum, the structure and nature of which have to be considered at the Planck distance $[5,32,36]$. This question is closely related to the structure of the leptons and other fundamental particles on the same distances. According to [37-39] the center of an electron is extreme maximon, that is the quantum nonsingular object creating round itself an extreme Kerr-Newman metric. It has spin $s=1 / 2$ and approximately Planck mass, charge and radius. For most observed phenomena involving photons it is possible to give the following interpretation of their propagation in vacuum. In the photon propagation the middleordered (in time and space) alternate spins flip occurs (during the Planck time $T_{P}$ for each flip, see Fig.4a) of virtual vacuum extreme maximons, which creates the effect of the spin wave, and in "macroscopic scale" produce manifestation the corpuscular-wave properties of photons. However due to the vector dominance also exhibited by the photons, their propagation in vacuum can be associated also with other, more complex virtual processes.

\section{THE YOUNG'S INTERFERENCE EXPERIMENT}

It is known that when problem of wave-particle duality is discussed, then for example in experiment such as the Young's experiment, to explain the wave properties of particles having mass, the wave function in the coordinate representation is attracted. The similar picture of diffraction and interference observed with the light is explained, appealing to the classical electrodynamics based on Maxwell's equations. So, in the case of Young's experiment the explanation of the interference result is reduced to establishment of the phases difference of two monochromatic waves emitted by the slits 1 and 2 (see Fig. 4b), entering the observation point $P$ on the screen.

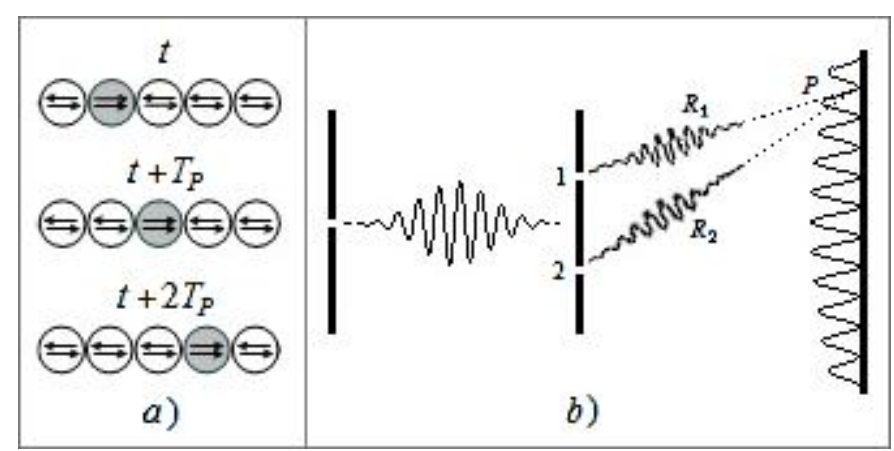

Fig. 4. Propagation of the photon in space: a) spins flip wave in physical vacuum at Planck distances; b) penetration of the wave function of a photon at a time through two slits in Young's experiment.

A similar phase $\varphi=\mathbf{k r}-k c t$ is available in each term of the photon wave function (1). If the radiation is more or less monochromatic, then in the expression for the probability density (3), obviously, arises the member proportional to the cosine of the phases difference $\delta=\varphi_{1}-\varphi_{2}=k\left(R_{1}-R_{2}\right)$ of these two waves (emitted by the slits 1 and 2). This also provides an explanation for the occurrence of interference fringes, similarly to the explanation of classical electrodynamics.

Thus, introducing in consideration the photon wave function in the coordinate representation, we have the opportunity to explain the wave phenomena on a uniform basis for all quantum particles. This especially becomes relevant when an experiment involving photons emitted obviously alone (for the first time single photons have been reliably fixed in [40]).

\section{CONCLUSION}

The results of our modeling of photon wave packet propagation allow to illustrate the possibility of a singlephoton approach to the description of electromagnetic phenomena. In particular, it appears that those aspects of interference and diffraction such as the interference pattern of Young's double-slit experiment, which were described in the 
language of classical electrodynamics, obviously can be described in the language of quantum mechanics without the involvement of the apparatus of second quantization of the electromagnetic field. This significantly expands the scope of "ordinary" quantum mechanics and considerably reduces the problem of wave-particle duality in the present level of our knowledge. However, in our view, the photon is a quasiparticle actually, and light is a result of the propagation of a spin-flip wave in physical vacuum, the structure and nature of which have to be considered at the Planck distance. Perhaps, this circumstance will allow to look from the new point of view at the such problems as the quantum entanglement of particles and their nonlocality of the interactions.

\section{REFERENCES}

[1] A.P. Davydov, "Jevoljucija v prostranstve i vo vremeni volnovogo paketa fotona femtosekundnogo izluchenija s tochki zrenija kvantovoj mehaniki", in Tezisy dokl. XLIII vnutrivuz. nauch. konf. prepodavatelej MaGU "Sovremennye poblemy nauki i obrazovanija", pod red. P.Ju. Romanova i E.M. Razinkinoj. Magnitogorsk: Izd-vo Magnitogorsk. gos. un-ta, pp. 269-270, 2005.

[2] A.P. Davydov, "Modelirovanie rasprostranenija v trehmernom prostranstve volnovogo paketa fotona", in Aktual'nye poblemy sovremennoj nauki, tehniki i obrazovanija: materially $73-\mathrm{j}$ mezhdun. nauch.-tehnich. konf. Magnitogorsk: Izd-vo Magnitogorsk. gos. tehn. un-ta im. G.I. Nosova, vol. 3, pp. 133-137, 2015.

[3] A.P. Davydov, T.P. Zlydneva, "Odnofotonnyj podhod k modelirovaniju korotkoimpul'snogo lazernogo izluchenija", in Vestnik nauki i obrazovanija Severa-Zapada Rossii: jelektronnyj zhurnal, vol. 1, № 4, 2015. URL: http://vestnik-nauki.ru/.

[4] A. Davydov, T. Zlydneva, "Modeling of short-pulse laser radiation in terms of photon wave function in coordinate representation", in press.

[5] A.P. Davydov, "Volnovaja funkcija fotona $\mathrm{v}$ koordinatnom predstavlenii”: monografija. Magnitogorsk: Izd-vo Magnitogorsk. gos. techn. un-ta im. G.I. Nosova, 2015. 180 p.

[6] L. Landau and R. Peierls, "Quantenelectrodynamik im Konfigurationsraum”, Zeit. F. Phys, vol. 62, pp. 188-198, 1930.

[7] H.A. Kramers, Quantum Mechanics, Amsterdam: North- Holland, 1958 (original edn 1937).

[8] T.D. Newton and E. P Wigner. "Localized states for elementary particles", Reviews of Modern Physics, vol. 21, pp. 400-406, 1949.

[9] D. Bohm, Quantum Theory, Constable, London, 1954.

[10] E. A. Power, Introductory Quantum Electrodynamics, Longmans, London, 1964.

[11] V.B. Berestetskii, E.M. Lifshitz, and L. P. Pitaevskii, Quantum electrodynamics, 2nd ed. (Pergamon Press Ltd., NY, 1982).

[12] A.I. Ahiezer and V. B. Beresteckij, Kvantovaja jelectrodynamika. M.: Nauka, 1981.

[13] V.G. Levich, Ju. A.Vdovin, and V. A. Mjamlin, Kurs teoreticheskoj fiziki, Tom II. M.: Nauka, 1971.

[14] R.Y. Chiao, P.G. Kwiat, and A.M. Steinberg, "Quantum non-locality in two-photon experiments at Berkeley", Quantum and Semiclassical Optics: Journal of the European Optical Society, Part B, vol. 7, № 3, pp. 259-278, 1995.

[15] M.O. Scully and M.S. Zubairy, Quantum Optics. Cambridge Univ. Press, 1997, Chs. 1 and 21.

[16] V.I. Fushhich and A.G. Nikitin, "O novyh i staryh simmetrijah uravnenij Maksvella i Diraka", W.I. Fushchych. Scientific Works, vol. 2. Kyiv: Editor Vyacheslav Boyko, 2000, pp. 233-278.

[17] A.K. Zvezdin, "Kvantovaja mehanika plenennyh fotonov, opticheskie microrezonatory, volnovody, fotonnye kristally”, Priroda, № 10, 2004, pp. 12-23.

[18] E.E. Zanimonskij and Ju.P. Stepanovskij, "Precessija spina fotonov i geometricheskie fazy", Visnik HNU, № 914, Serija Fizika, Vyp. 13, pp. 36-39, 2010.
[19] A.T. Gavrilin, "Ob amplitude verojatnosti mestopolozhenija fotona", Vestnik Nizhegorodskogo universiteta im. N.I. Lobachevskogo, № 6 (1), 2011, pp. 70-74.

[20] I. Bialynicki-Birula, "On the Wave Function of the Photon", Acta Phys. Pol. A, vol. 86, pp. 97-116, 1994.

[21] M. Mandel and E. Wolf, Optical coherence and quantum optics, Cambridge University Press, 1995.

[22] J. E. Sipe "Photon wave functions", Physical Review A, vol. 52, 1995, pp. $1875-1883$.

[23] A.P. Davydov, "Kvantovaja mehanika fotona", in Tezisy dokl. XXXIII nauch. konf. prepodavatelej MGPI "NAUKA I SHKOLA". Magnitogorsk: Izd-vo Magnitogorsk. gos. ped. in-ta, pp. 206-207, 1995.

[24] I. Bialynicki-Birula. "The Photon Wave Function", in Coherence and Quantum Optics VII, edited by J. H. Eberly, L. Mandel, and E. Wolf (Plenum Press, New York, 1996), pp. 313-323.

[25] I. Bialynicki-Birula, "Photon Wave Function", in Progress in Optics, edited by E. Wolf (North-Holland, Elsevier, Amsterdam, 1996), vol. XXXVI, pp. 248-294.

[26] M. Hawton, "Photon wave functions in a localized coordinate space basis", Phys. Rev. A, vol. 59, pp. 3223-3227, 1999.

[27] A.P. Davydov, Volnovaja funkcija fotona $\mathrm{v}$ koordinatnom predstavlenii", in Vestnik MaGU: Periodicheskij nauchnyj zhurnal, Vyp. 5, Estestvennye nauki. Magnitogorsk: Izd-vo Magnitogorsk. gos. un-ta, pp. 235-243, 2004.

[28] B.J. Smith and M.G. Raymer, "Photon wave functions, wave-packet quantization of light, and coherence theory," New J. Phys., vol. 9, pp. 414-448, 2007.

[29] J. Cugnon, "The Photon Wave Function", Open Journal of Microphysics, vol. 1, № 3, pp. 41-52, 2011.

[30] E. Brainis, "Quantum imaging with $N$-photon states in position space", Optics Express, vol. 19(24), pp. 24228-24240, 2011.

[31] P. Saari, "Photon localization revisited," in Quantum Optics and Laser Experiments, Edited by S.Lyagushyn, S., InTech, Open Access Publisher, Croatia , pp. 49-66, 2012.

[32] A.P. Davydov, "Kvantovaja mehanika fotona: volnovaja funkcija v koordinatnom predstavlenii", Jelektromagnitnye volny i jelektronnye sistemy, vol. 20, № 5, pp. 43-61, 2015.

[33] R. Mignani, E. Recami, and M. Baido, "About a Diraclike Equation for the Photon, According to Ettore Majorana", Left. Nuovo Cimento, vol.11, № 12, pp. 568-572, 1974.

[34] A.P. Davydov, "O postroenii special'noj teorii otnositel'nosti (STO) iz simmetrii prostranstva i vremeni bez postulatov STO", Jelekrtromagnitnye volny i jelektronnye sistemy, vol. 8, № 1, pp. 49-58, 2003.

[35] A.P. Davydov, "Kurs lekcij po kvantovoj mehanike. Matematicheskij apparat kvantovoj mehaniki": Ucheb. posobie. Magnitogorsk: Izd-vo Magnitogorsk. gos. tehn. un-ta im. G.I. Nosova, 2014, 188 p.

[36] A.P. Davydov, "Foton kak kvazichastica pri vozbuzhdenii spinovoj volny v fizicheskom vakuume na plankovskih rasstojanijah", in Tezisy dokl. XLIV vnutrivuz. nauch. konf. Prepodav. MaGU "Sovremennye problem nauki i obrazovanija". Magnitogorsk: Izd-vo Magnitogorsk. gos. un-ta, p. 174, 2006.

[37] A.P. Davydov, "Novye kvantovye ob\#ekty kosmomikrofiziki jelementarnye bessinguljarnye chernye dyry - kak sledstvie KJeD i OTO", in Sb. nauch. trudov "Fundametal'nye prikladnye issledovanija". Magnitogorsk: Izd-vo MGPI, pp. 22-41, 1997.

[38] A.P. Davydov, "Vozmozhnost' kvantovyh bessinguljarnyh chernyh dyr $\mathrm{s}$ plankovskimi parametrami i jekstremal'noj metrikoj $\mathrm{v}$ fizike $\mathrm{i}$ kosmologii”, Jelektromagnitnye volny i jelektronnye sistemy, vol. 3, № 2, pp. 67-78, 1998.

[39] A.P. Davydov, "Jekstremal'nye maksimony, struktura fundamental'nyh chastic, KJeD, OTO i RTG A.A. Logunova", Jelektromagnitnye volny i jelektronnye sistemy, vol. 6, № 5, pp. 4-13, 2001.

[40] J.F. Clauser, "Experimental distinction between the quantum and classical field theoretic predictions for the photo-electric effect", Phys. Rev. D, vol. 9, pp. 835-860,1974. 